\title{
Nonlinear supratransmission
}

\author{
F Geniet and J Leon \\ Physique Mathématique et Théorique, CNRS-UMR 5825, 34095 Montpellier, France
}

Received 7 January 2003

Published 22 April 2003

Online at stacks.iop.org/JPhysCM/15/2933

\begin{abstract}
A nonlinear system possessing a natural forbidden band gap can transmit energy of a signal with a frequency in the gap, as recently shown for a nonlinear chain of coupled pendulums (Geniet and Leon 2002 Phys. Rev. Lett. 89 134102). This process of nonlinear supratransmission, occurring at a threshold that is exactly predictable in many cases, is shown to have a simple experimental realization with a mechanical chain of pendulums coupled by a coil spring. It is then analysed in more detail. First we go to different (nonintegrable) systems which do sustain nonlinear supratransmission. Then a Josephson transmission line (a one-dimensional array of short Josephson junctions coupled through superconducting wires) is shown to also sustain nonlinear supratransmission, though being related to a different class of boundary conditions, and despite the presence of damping, finiteness, and discreteness. Finally, the mechanism at the origin of nonlinear supratransmission is found to be a nonlinear instability, and this is briefly discussed here.
\end{abstract}

\section{Introduction}

It has recently been demonstrated that, in addition to energy spectral localization (the FermiPasta-Ulam recurrence phenomenon [1]) and energy spatial localization (soliton generation from initial data [2-5]), a nonlinear chain of oscillators possesses another striking fundamental property called nonlinear supratransmission [6]. This phenomenon was shown to occur in the nonlinear sine-Gordon chain, which possess a natural forbidden band gap, when it is subjected to irradiation at a frequency in the stop gap. While in a linear chain the signal would exponentially vanish in the medium, it does not do so in the nonlinear case if its amplitude exceeds a threshold value.

In [6] the irradiation of the medium was modelled by prescribing the boundary value at one end of the chain. It is worth mentioning that nonlinear transmission does occur also in the case of a true wave scattering, namely when a monochromatic plane wave scatters onto a nonlinear medium with a frequency in the gap [7].

In the case of the sine-Gordon model, the threshold can be predicted exactly by invoking the static one-breather solution [6]. In short, energy penetrates the medium as soon as the amplitude $A$ of the harmonic driving, at a frequency $\Omega$ in the gap, exceeds the maximum 
amplitude of the static breather of frequency $\Omega$. This energy then travels through the medium by means of nonlinear localized excitations (kinks, breathers, solitons).

The purpose of this paper is, after recalling results of [6], to display experimental results on the mechanical chain of pendulums and to discuss the generality of our result by exploring extensions to different situations. We shall in particular explore the robustness of nonlinear supratransmission when the medium experiences damping, different nonlinearities, and different classes of boundary values.

The next section is devoted to a short recap of the results published in [6] and it is intended to settle the formalism and the basic facts about nonlinear supratransmission. The model is the sine-Gordon chain subjected to a Dirichlet condition at the origin on a vanishing initial background (pendulums at rest). Then section 3 relates the experiment carried out with a mechanical chain of pendulums, coupled by means of a coil spring, and which is forced at one end by a periodic torque. A systematic exploration of the chain response in a frequency range within the gap shows spectacular agreement with the theory. In section 4 we describe numerically the characteristics of the breathers generated in the sine-Gordon chain and discover some simple relations between the parameters of the emitted breather and those of the boundary driving. Section 5 deals with the energy transmitted by the nonlinear medium as a function of the driving amplitude at a given frequency in the gap. Particular emphasis will be put on the effectiveness of the effect in different, not necessarily integrable, cases. The nonlinear instability which is the generating mechanism of nonlinear supratransmission is briefly discussed in section 6. Although the mathematical analysis is still to be constructed, we propose a quite simple illustration of the process by means of a perturbative analysis of the sine-Gordon system driven close to a breather mode.

As another domain of study, we consider in section 7 the sine-Gordon model for Neumann conditions at the boundary (the derivative at the origin is prescribed). This is a model for a chain of short Josephson junctions whose first one is subjected to an external AC current. By using numerical simulations, the process of nonlinear supratransmission is shown to hold, and the threshold of energy transmission to obey a similar simple rule. Remarkably, in this case the energy flows by means of kinks (or antikinks) and not by means of breathers (or kink-antikink pairs). This is an interesting issue as regards applications, as kinks are the objects that have an experimental signature (through the Josephson current).

\section{The sine-Gordon chain}

\subsection{Generalities}

The following normalized discrete sine-Gordon chain of $N$ locally damped coupled oscillators $u_{n}(t)$ :

$$
\ddot{u}_{n}-c^{2}\left(u_{n+1}-2 u_{n}+u_{n-1}\right)+\sin u_{n}=-\gamma(n) \dot{u}_{n}, \quad n=1, \ldots, N,
$$

is subjected to the boundary value at the origin (Dirichlet condition)

$$
u_{0}(t)=A \sin \Omega t,
$$

acting on a medium initially at rest, namely

$$
u_{n}(0)=0, \quad \dot{u}_{n}(0)=0 .
$$

In this section the damping coefficient $\gamma$ is used to model a semi-infinite chain by an absorbing boundary. More precisely, we take $u_{N+1}(t)=0$ with

$$
\begin{array}{ll}
0 \geqslant n \leqslant m: & \gamma(n)=0, \\
m<n \leqslant N: & \gamma(n)=a\left[1+\tanh \left(\frac{2 n-m-N}{2 b}\right)\right],
\end{array}
$$


where the parameter $b$ is adjusted to have a damping factor $\gamma(n)$ varying slowly from almost 0 to almost $2 a$ on the last $(N-m)$ particles. A typical experiment will have e.g. $N=100$, $m=60, a=0.5$, and $b=3$. Note that in section 7 we shall use instead the reflective boundary condition $u_{N+1}=u_{N}$ for a homogeneous damping $\gamma(n)=$ cste for all $n$.

Equation (1) is considered as a second-order ordinary differential system for the $N$ coupled oscillators $u_{n}(t)$. This system is then solved with the subroutine dsolve of the MAPLE software package which uses a Fehlberg fourth-fifth-order Runge-Kutta method.

\subsection{Forcing in the gap}

The linear dispersion relation $\omega(k)$ of the chain is

$$
\omega^{2}=1+2 c^{2}(1-\cos k) .
$$

For a driving boundary (2) with the frequency in the forbidden band gap (FBG), namely $\Omega<1$, a linear chain would sustain the solution (evanescent wave)

$$
A \sin (\Omega t) \exp [-\lambda n] \text {. }
$$

The parameter $\lambda$ is given from the dispersion relation (5) written for $\omega=\Omega<1$ and $k=i \lambda$, namely

$$
\lambda=\operatorname{arccosh}\left(1+\frac{1-\Omega^{2}}{2 c^{2}}\right) .
$$

In the nonlinear case, in order to fit the boundary condition (2), the approximate solution (exact in the continuous limit) is given instead by the static breather

$$
u_{b}(n, t)=4 \arctan \left[\frac{\lambda c \sin (\Omega t)}{\Omega \cosh \left(\lambda\left(n-n_{0}\right)\right)}\right],
$$

where the breather centre $n_{0}$ solves

$$
A=4 \arctan \left[\frac{\lambda c}{\Omega \cosh \left(\lambda n_{0}\right)}\right] .
$$

The above equation expresses that the static breather fits the driving field (2) by adjusting its position so as to match the amplitude $A$. The spectrum of the breather signal does not match exactly the monochromatic forcing (2) and it adapts by sending phonons at third, fifth, etc, harmonic frequencies.

The above assertion is checked by performing two simulations of (1) at a given forcing frequency, say $\Omega=0.8$, and a given amplitude, say $A=2$, in one case with the harmonic forcing (2), and in the other case with the breather-like forcing $u_{b}(0, t)$ where the value of $n_{0}$ is calculated from (9) with $A=2$. To avoid initial shock we also set initial velocities matching those of the static breather. Then we evaluate by Fourier transformation the spectra of one particle of the chain in both cases (we have selected particle 50 on a chain of 100 pendulums). The result is displayed in figure 1 where we see that phonons at frequency $3 \Omega$ are indeed emitted for harmonic forcing while no phonon appears for a breather-like forcing.

The small contribution at frequency 1 in figure 1(a) represents a collective motion of the pendulums generated by the initial shock resulting from the inadequacy of the initial velocities compared with the boundary value.

\subsection{Bifurcation threshold}

The adjustment of the breather centre $n_{0}$ provided by (9) not always has a solution. Indeed it works up to the maximum value $A=A_{s}$ of the breather amplitude realized for $n_{0}=0$. 

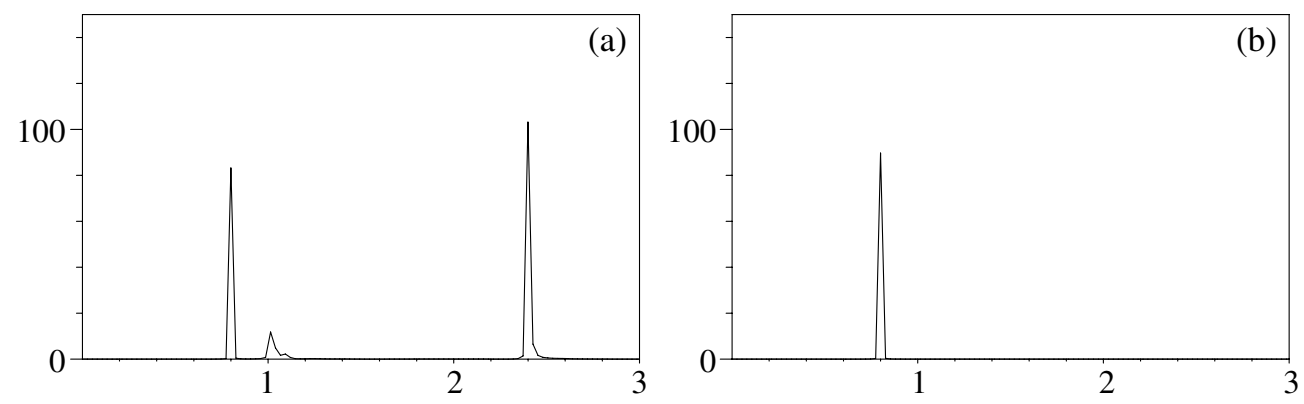

Figure 1. Spectra of particle 50 subjected to (a) harmonic boundary driving at frequency 0.8 and amplitude 2 and to (b) breather boundary driving at frequency 0.8 .

Beyond this threshold, for a driving boundary with $A>A_{s}$, we have shown in [6] that nonlinear supratransmission occurs, i.e. the medium starts to transmit energy by means of nonlinear mode generation (breathers and kink-antikink pairs). From (8), the threshold $A_{s}$ reads as the following function of the frequency $\Omega$ :

$$
A_{s}=4 \arctan \left[\frac{c}{\Omega} \operatorname{arccosh}\left(1+\frac{1-\Omega^{2}}{2 c^{2}}\right)\right],
$$

which has the approximate value

$$
A_{s} \sim 4 \arctan \left[\frac{\sqrt{1-\Omega^{2}}}{\Omega}\right]
$$

which would hold for the continuous sine-Gordon equation obtained in the limit $1 / c \rightarrow 0$. Note that the maximum difference (when $\Omega$ varies in $[0,1]$ ) between the above two expressions is already just $1 \%$ for $c=1$ and goes down to $0.01 \%$ for $c=10$, the value that we have used in most of the numerical simulations.

This qualitative definition of a bifurcation threshold can be checked by means of numerical simulations of (1) with the boundary condition (2) by varying, at given frequency $\Omega$, the amplitude $A$ around the above value $A_{s}=1.803$ (for $c=4$ ). There are many means for determining that nonlinear supratransmission has appeared, a simple one being via the observation of the motion of one particle of the chain. As an example, we display in figure 2 the motion of particle 60 of a chain of 200 particles driven at frequency 0.9 at amplitudes $A=1.78$ (no supratransmission) and $A=1.79$ (supratransmission) for a coupling factor $c^{2}=16$. Each large oscillation in the second figure corresponds to a breather passing by. Two of them are generated and cross site 60 at times 120 and 160. The small oscillations seen between the humps are the harmonic phonons, mainly of frequency $3 \Omega$.

For illustration we show in figure 3 a picture of the oscillator amplitude $u_{n}(t)$ at given time (here $t=120$ ) in the case of nonlinear supratransmission obtained for $A=1.8$ and $\Omega=0.9$. A first breather propagates to the right while a second one is just being generated near the origin. Note the amplitude of the breather with respect to that of the driving boundary.

By a systematic exploration of the chain response we plotted figure 4, obtained for 200 particles with a coupling $c^{2}=100$ (some experiments have actually been carried out with smaller coupling and fewer points, to shorten computation times) for a typical time of 200 (for frequencies close to the gap value 1 , time had to be increased to 500). The points on figure 4 are obtained with an absolute precision of $10^{-2}$ for the amplitude $A$. They are compared to the theoretical threshold expression (10) (continuous curve). 

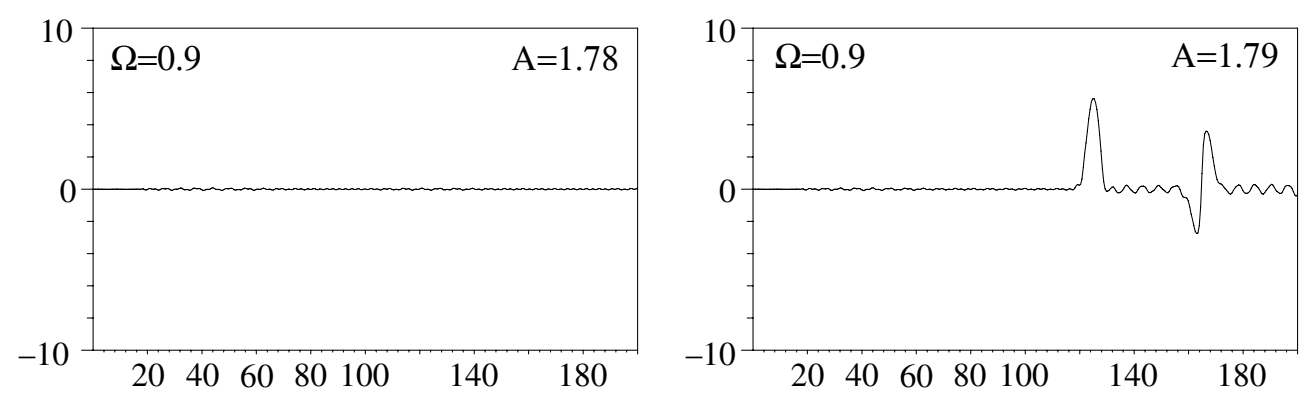

Figure 2. Representation of $u_{n}(t)$ as a function of time for $n=60$ in the case $\Omega=0.90$ for two amplitudes.

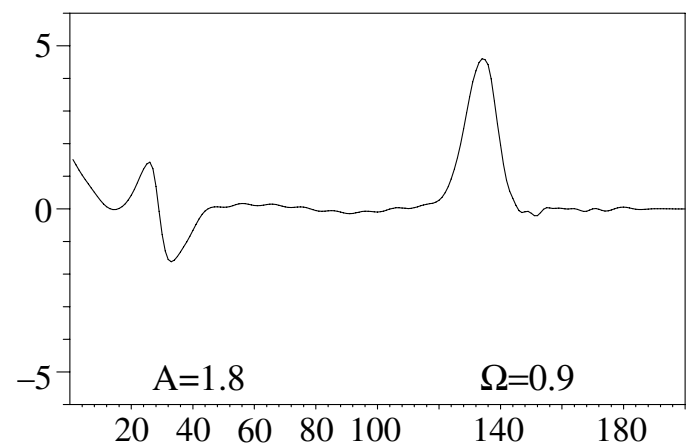

Figure 3. A plot of $u_{n}(t)$ as a function of $n$ at $t=120$.

Figure 4 shows excellent agreement with formula (10) apart for some discrepancies starting below 0.33 and 0.18 . This results from the driving which, thanks to the nonlinearity, generates phonons at multiple frequencies (here third, fifth, etc). If these frequencies lie in the phonon band, the phonons move away from the boundary and have no effect on the forcing. If however they lie in the FBG, the related phonons do not propagate (which we call phonon quenching) and stick on the boundary where they make a contribution to the driving. This effect indeed disappears when driving the system (1) with the exact breather boundary value $u_{b}(0, t)$ for which we have checked that nonlinear supratransmission never occurs at an amplitude $A<A_{s}$, while it is already occurring at $A=A_{s}$ (or for very small deformation of the perfect breather).

\section{Experiments on a mechanical chain}

Our purpose here is to show that the process of nonlinear supratransmission can be easily realized experimentally on a chain of coupled pendulums as depicted in figure 5. This chain has been built following Remoissenet [5]. The pendulums rotate freely around a piano wire stretched between two supports; they are coupled together by a coil spring tightened to each pendulum by a screw.

Such a chain of 48 pendulums is driven by an electrical engine steered by a generator of sinusoidal tension. Upon varying the frequency, at low amplitude, in the phonon band, we can determine the parameters of the chain (coupling constant $\sigma^{2}$ and angular eigenfrequency $v_{0}$ ) by measuring the wavelength $2 \pi / k$ of the wave produced (in units of the number of pendulums). 


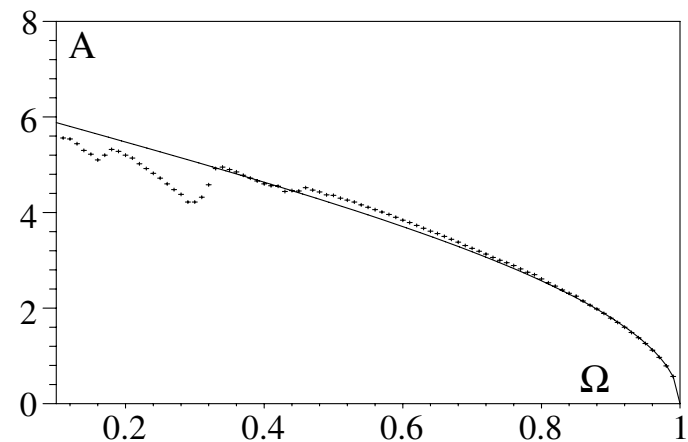

Figure 4. The bifurcation diagram in the $(A, \Omega)$ plane. The solid curve is the threshold expression (10). The crosses indicate the lowest value of $A$ for which nonlinear supratransmission is seen in numerical simulations.

By comparison to the dispersion relation of the linear chain

$$
v^{2}=v_{0}^{2}+2 \sigma^{2}(1-\cos k)
$$

we determined the following parameter values:

$$
\nu_{0}=15 \mathrm{~Hz}, \quad \sigma=32 \mathrm{~Hz} .
$$

Then in a time normalized to the eigenfrequency, we have the dispersion relation (5) with

$$
c=\frac{\sigma}{v_{0}}=2.13
$$

which is the fundamental parameter of the model, entering, in particular, expression (10) for the threshold amplitude for nonlinear supratransmission. This is the formula that we want here to confront to experiments.

We proceed with a systematic exploration of the chain response to a signal frequency in the gap (of value less than $v_{0}$ ). The method consists in increasing slowly, at fixed frequency, the amplitude of the driving, up to the time when a nonlinear mode is seen to be generated (a picture of a breather generated at a frequency of 0.85 in normalized units is displayed in [6]). Repeating three times, for each driving frequency value, the measurements of the driving amplitude that generates a nonlinear mode, we eventually obtain figure 6 which displays the measured threshold amplitude in terms of the signal frequency (normalized). There, the full curve is the function $A_{s}(\Omega)$ given in (10) with $c=2.13$.

Despite the small number of pendulums, inducing reflection at the open end, the inherent damping, and other mechanical imperfections, the experiments provide a spectacular realization of the theoretical threshold prediction.

\section{Characteristics of the emitted breathers}

One important issue concerns the nature and the characteristics of the nonlinear modes which propagate in the medium. In the first instance, we have to check that the emitted structures correctly match the moving breather, at velocity $v<1$, which it is convenient to write as

$$
u_{v}(n, t)=4 \arctan \left[\frac{r \sin \left(\frac{1}{\sqrt{1+r^{2}}} \frac{t-v n / c}{\sqrt{1-v^{2}}}\right)}{\cosh \left(\frac{r}{\sqrt{1+r^{2}}} \frac{n / c-v t)}{\sqrt{1-v^{2}}}\right)}\right] \text {. }
$$






Figure 5. A sketch of the mechanical chain.



Figure 6. Experimental values of the nonlinear supratransmission threshold compared to expression (10).

By choosing correctly the two parameters $r$ and $v$ which represent respectively the amplitude and the group velocity of the breather, together with the space and time origins of the solution $u_{v}\left(n-n_{0}, t-t_{0}\right)$, one can match it to the asymptotic numerical solution with high precision. This enables us to identify correctly the emitted breathers, to determine their characteristics with great accuracy, and to observe their possible disintegration into kink-antikink pairs.

The results of such estimations are displayed in figure 7 obtained as follows. For each driving frequency $\Omega$, we have driven the system at the threshold amplitude $A_{s}(\Omega)$ and then determined the parameters $\{r, v\}$ of the emitted breathers (a given experiment at threshold driving produces repeatedly the same breather). Then the amplitude of the propagating breather is plotted as a function of the amplitude of excitation $A_{s}(\Omega)$ for various frequencies. Similarly, figure 8 shows the group velocity of the breather as a function of $A_{s}(\Omega)$.

Both figures 7 and 8 correspond to a region of the driving frequency close to the gap $0.87 \leqslant \Omega \leqslant 1$. For lower frequencies the emitted breathers, when they occur, are unstable and decay into kink-antikink pairs (this will be discussed later). The main conclusion here is that there exists a linear relation between the amplitude $A_{b}$ (and the velocity $v$ ) of the breather generated and the driving amplitude at threshold $A_{s}$. So far we have no theoretical interpretation of these observations.

Another characteristic of the breather is its proper frequency $\omega_{b}$, related to the apparent pulsation period $T$ by the usual (relativistic) relation

$$
\omega_{b}=\frac{1}{\sqrt{1+r^{2}}}=\frac{2 \pi}{T \sqrt{1-v^{2}}} .
$$






Figure 7. The amplitude of the emitted breathers in terms of the amplitude of excitation, just above the emission threshold.

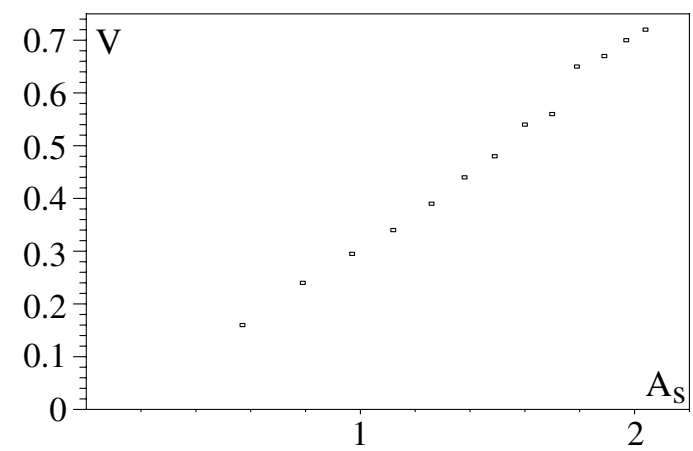

Figure 8. The group velocity of the emitted breathers, as a function of the amplitude of excitation, just above the emission threshold.

This frequency is plotted in figure 9 as a function of the driving frequency $\Omega$ and, again, we note a nontrivial linear dependence.

We now turn to the stability of the emitted breathers which, for $\Omega \leqslant 0.88$, decay into kink-antikink pairs. This can be qualitatively described by studying the binding energy $W$, given by

$$
W=2 E_{k}-E_{b}=\frac{16}{\sqrt{1-v^{2}}}\left(1-\sqrt{\frac{r^{2}}{1+r^{2}}}\right),
$$

where $E_{k}$ is the single kink energy and $E_{b}$ the breather energy [5].

We have evaluated the binding energy $W$ of the breathers produced in the simulations (by plugging into (17) the measured values of $r$ and $v$ ); the result is plotted in figure 10 in terms of the frequency $\Omega$ of the driving boundary. This figure shows that the binding energy of the breather decreases with the driving frequency, and goes to zero for $\Omega \lesssim 0.88$. Thus the breather decay in the frequency range $[0,0.8]$ is well understood from formula (17). 




Figure 9. The proper frequency of the emitted breathers, as a function of the driving frequency $\Omega$, just above the emission threshold.

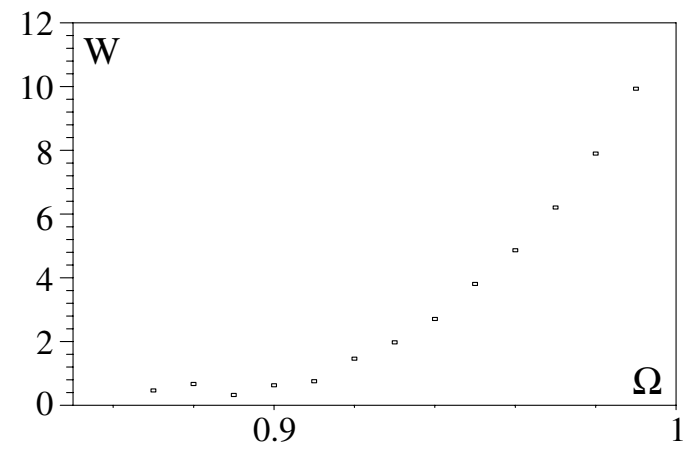

Figure 10. The binding energy of the emitted breathers, as a function of the frequency of excitation, just above the emission threshold.

\section{Energy transmission}

\subsection{Basic expression}

Nonlinear supratransmission is a process where a large amount of energy flows through the medium. Our purpose here is to evaluate numerically this energy for amplitudes around the threshold value. The theoretical expression for the energy flow is calculated hereafter for a generic nonlinearity deriving from a potential energy $V\left(u_{n}\right)$ (the sine-Gordon case (1) corresponds to $\left.V\left(u_{n}\right)=1-\cos u_{n}\right)$.

From the energy density,

$$
H_{n}=\frac{1}{2} \dot{u}_{n}^{2}+\frac{c^{2}}{2}\left(u_{n+1}-u_{n}\right)^{2}+V\left(u_{n}\right), \quad n=1, \ldots, \infty,
$$

and the evolution equation follows the conservation law

$$
\frac{\partial}{\partial t} H_{n}+\left(J_{n+1}-J_{n}\right)=0,
$$

with the current

$$
J_{n}=-c^{2} \dot{u}_{n}\left(u_{n}-u_{n-1}\right) .
$$


Incorporating the potential energy resulting from the coupling of the first particle $u_{1}$ to the boundary $u_{0}$, the total energy of the system reads

$$
E=\sum_{n=1}^{\infty} H_{n}+\frac{c^{2}}{2}\left(u_{1}-u_{0}\right)^{2} .
$$

In our case $u_{0}(t)$ is the driving (2) and the chain is supposed infinite with $u_{n}(t) \rightarrow 0$ as $n \rightarrow \infty$.

Upon taking the time derivative, with help of the conservation law, and using the assumed asymptotic $J_{n} \rightarrow 0$ for large $n$, we arrive eventually at

$$
\frac{\partial}{\partial t} E=c^{2} \dot{u}_{0}\left(u_{0}-u_{1}\right)
$$

Hence the total energy injected into the medium during time $T$ reads

$$
E=c^{2} \int_{0}^{T} \mathrm{~d} t \dot{u}_{0}(t)\left[u_{0}(t)-u_{1}(t)\right] .
$$

Choosing for $T$ an integer multiple of the period of excitation makes this energy vanish identically in the linear case if the driving frequency falls in the FBG.

\subsection{Numerical simulations}

In the nonlinear case, expression (23) is computed numerically for a chain of 60 particles with a coupling parameter $c^{2}=16$ and an absorbing end working over the last 40 particles. For a driving frequency 0.9 and amplitudes running from 1.5 to 2.0, we obtain figure 11 where the bifurcation is seen to occur for $A=1.80$, the value predicted by formula (10). This simulation has been run for frequencies in the range [0.2,0.9], with comparable expected results.

Our approach stems from the existence of a breather solution of the model equation, allowing us to determine the threshold amplitude. It is thus worth considering whether the process is robust against nonintegrability.

To give a partial yet instructive answer to this question, we have performed numerical simulations of two nonintegrable evolutions in the same class, i.e. with Hamiltonian (18).

First the Taylor truncated sine-Gordon expansion (the fifth order is kept to ensure a confining potential at large $u_{n}$ ) reads as for the nonlinear Klein-Gordon chain:

$\ddot{u}_{n}-c^{2}\left(u_{n+1}-2 u_{n}+u_{n-1}\right)+u_{n}-\frac{1}{3 !} u_{n}^{3}+\frac{1}{5 !} u_{n}^{5}=0, \quad n=1, \ldots, N$.

This system is solved with the boundary driving (2), and the energy (23) is computed for the same parameter values as for figure 11. The result is displayed in figure 12 .

By scanning the frequency range in the gap, we have found that the process occurs down to $\Omega=0.7$ and then disappears. We suspect that for such a polynomial potential energy, at high forcing amplitude, the incoming wave sees an almost parabolic potential, while for low-amplitude driving the incoming wave does feel the actual structure of the local potential.

Another interesting nonintegrable Hamiltonian evolution where the local potential has a periodic structure is the double sine-Gordon chain:

$\ddot{u}_{n}-c^{2}\left(u_{n+1}-2 u_{n}+u_{n-1}\right)+\frac{1}{3}\left[\sin u_{n}+\sin 2 u_{n}\right]=0, \quad n=1, \ldots, N$.

Once again we have solved this system in the same situation as before and obtained the sudden energy flow of figure 13. In this case the process holds for any frequency (tested from $\Omega=0.2$ ) just as in the sine-Gordon case.

Thus we have seen that in those two cases nonlinear supratransmission does work, which is a strong indication that it is a generic nonlinear process. An interesting question is that of the mechanism that generates nonlinear supratransmission, which is discussed now. 




Figure 11. The energy $E$ injected in the sine-Gordon nonlinear chain for $T=140$ as a function of the driving amplitude $A$.

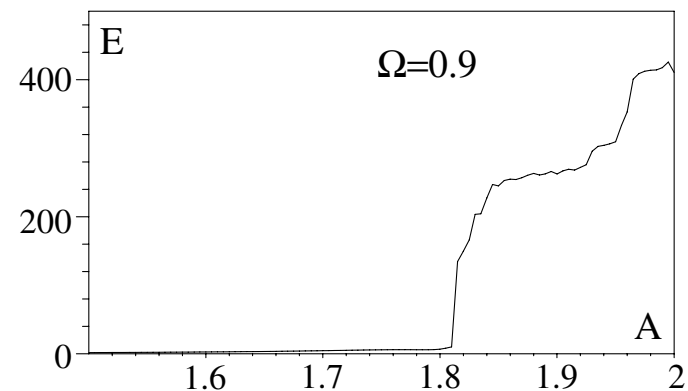

Figure 12. Energy in the Klein-Gordon chain (24).

\section{Generating instability}

The process at the origin of nonlinear supratransmission is a nonlinear instability. Although it is not yet fully understood, we discuss here some of its relevant aspects.

To that end we explore the properties of the sine-Gordon chain (1) subjected to initial boundary value conditions that precisely mimic the breather $b_{n}(t)$ centred at $n_{0}=0$ as defined in (8), which it is convenient to write as

$$
\begin{aligned}
b_{n}(t) & =4 \arctan \phi_{n}(t), \\
\phi_{n}(t) & =\frac{\lambda c}{\Omega} \frac{\sin \Omega t}{\cosh \lambda n} .
\end{aligned}
$$

Note that this is an exact solution in the continuous limit $1 / c \ll 1$ and $\left\{x=n / c, \lambda c=\kappa, \Omega^{2}=\right.$ $\left.1-\kappa^{2}\right\}$.

In order to study the behaviour at the threshold, we impose the boundary value

$$
u_{0}(t)=(1+\epsilon) b_{0}(t)
$$

and use the compatible initial data

$$
u_{n}(0)=(1+\epsilon) b_{n}(0), \quad \dot{u}_{n}(0)=(1+\epsilon) \dot{b}_{n}(0) .
$$

The boundary value at the other end of the chain $(n=N)$ can be taken as an absorbing end to simulate the infinite line or as the breather value $b_{N}(t)$, e.g. to check the accuracy of the solution.

The parameter $\epsilon$ measures the departure from the exact solution. We are of course interested in what happens when $\epsilon$ is positive. For $\epsilon=0$ we simply generate the approximate 


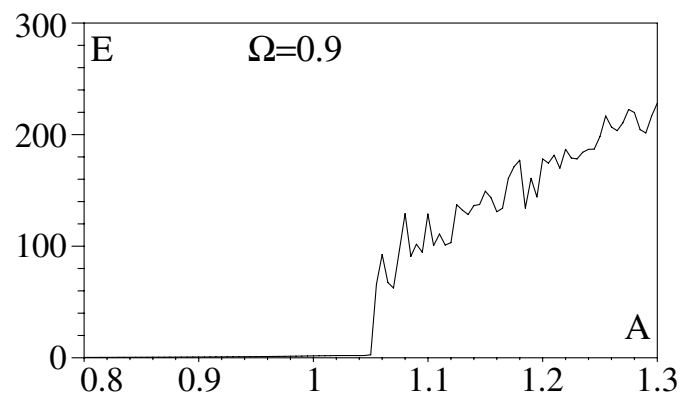

Figure 13. Energy in the double sine-Gordon chain (25).

solution $b_{n}(t)$ which in the numerical simulations is marginally stable (and would indeed be stable for a breather centred in $n_{0}<0$ ).

In the case $\epsilon>0$, the numerical simulations of the sine-Gordon model immediately generate nonlinear supratransmission. From the initial boundary value problem, it is natural to seek a solution as a perturbation of the breather in the form

$$
u_{n}(t)=b_{n}(t)+\epsilon \eta_{n}(t),
$$

which by (1) obeys at order 1 in $\epsilon$

$$
\ddot{\eta}_{n}-c^{2}\left(\eta_{n+1}-2 \eta_{n}+\eta_{n-1}\right)+C_{n} \eta_{n}=\epsilon D_{n} \eta_{n}^{2} .
$$

The variable coefficients $C_{n}(t)$ and $D_{n}(t)$ of this equation are given by

$C_{n}=\cos b_{n}=1-\frac{8 \phi_{n}^{2}}{\left(1+\phi_{n}^{2}\right)^{2}}, \quad D_{n}=\frac{1}{2} \sin b_{n}=2 \phi_{n} \frac{1-\phi_{n}^{2}}{\left(1+\phi_{n}^{2}\right)^{2}}$.

The initial boundary value problem that goes with (31) can be extended to results from (28), namely

$$
\eta_{0}(t)=b_{0}(t)
$$

with the related initial data. Then we observe the following fundamental facts:

- for $\epsilon<0$, the system (31) is stable, showing a long-period oscillatory behaviour;

- for $\epsilon>0$, the system is unstable, showing an exponential growth of the oscillations.

These observations are illustrated by figure 14 where we have computed the energy transmitted by the chain (31) according to formula (23), as a function of time (the points in time are chosen as integer multiples of the period $2 \pi / \Omega$ ). The parameters related to this experiment are $N=100, c=10$ and the energy has been calculated for ten points (up to $t=20 \pi / \Omega$ ).

We have also displayed results for the linear version of system (31) (read with $\epsilon=0$ ) which is marginally unstable, showing a linear growth of the oscillations. The exponential growth in the case $\epsilon>0$ is the signature of the instability which is the mechanism of nonlinear supratransmission. A mathematical approach to this instability for (31) will be the subject of future study.

\section{Josephson junction arrays}

\subsection{The model}

A Josephson junction behaves just like a single pendulum, the rotation amplitude being replaced by the phase difference between wavefunctions of Cooper pairs [8]. On connecting the 




Figure 14. The energy $E(T)$ given by (23) computed for the chain (31): (a) exponential growth for $\epsilon=0.005$; (b) linear growth for $\epsilon=0$; and (c) oscillations for $\epsilon=-0.005$. $E(T)$ varies here on the scale $\left[0,2 \times 10^{4}\right]$.

junctions in parallel with superconducting wires, the resulting model equation is just the sine-Gordon discrete system subjected to damping and constant torque [9]:

$$
\ddot{u}_{n}+\gamma \dot{u}_{n}+\sin u_{n}=J+c^{2}\left(u_{n+1}-2 u_{n}+u_{n-1}\right), \quad n=1, \ldots, N .
$$

Here, $\gamma$ is the constant damping along the array, $J$ is the normalized intensity of the applied current, and time has been normalized to the plasma frequency.

We consider here only the one-dimensional geometry, but it is worth mentioning the coupled arrays (ladders) revealed as a means to generate discrete breathers by using convenient initial conditions [10], with subsequent striking experimental realizations [11].

The applied current can have a DC component (the so-called bias) and an AC driving part. The problem that we are interested in is the behaviour of the above chain initially at rest and whose first junction only is subjected to AC driving at a frequency $\Omega$ in the gap and intensity $I$. Then the model results as (34) for $n>1$ and the bias $J=I_{0}$ together with the following relation for $n=1$ :

$$
\ddot{u}_{1}+\gamma \dot{u}_{1}+\sin u_{1}=c^{2}\left(u_{2}-u_{1}\right)+I_{0}+I \sin \Omega t .
$$

It is important to note that the above equation can be equivalently written as the discrete Neumann condition

$$
c\left(u_{1}-u_{0}\right)=-\frac{I}{c} \sin \Omega t,
$$

when the system (34) is assumed to hold also for $n=1$. This actually allows us to consider the continuous limit in order to determine a prediction for the threshold of nonlinear supratransmission.

\subsection{Bifurcation threshold prediction}

The continuous version of (34) and boundary value (36), for the variable $x=n / c$ and $c \rightarrow \infty$, reads as the system

$$
\begin{aligned}
& u_{t t}+\gamma u_{t}+\sin u=I_{0}+u_{x x}, \\
& \left.\partial_{x} u\right|_{x=0}=B \sin \Omega t,
\end{aligned}
$$

where we have defined $B=-I / c$. This continuous version corresponds to a long Josephson junction whose extremity $x=0$ is subjected to external microwave irradiation, the amplitude $B$ being then related to the external magnetic field intensity [12]. This is a Neumann boundary condition for the sine-Gordon continuous equation (37). 
The system will then adapt to the breather derivative at the boundary centred at $-x_{0}$; that is,

$$
\left.\partial_{x} u_{b}\right|_{x=0}=4 \frac{\kappa^{2}}{\Omega} \frac{\sin \Omega t \sinh \kappa x_{0}}{\cosh ^{2} \kappa x_{0}+\left(\kappa^{2} / \Omega^{2}\right) \sin ^{2} \Omega t},
$$

where now the continuous version of the dispersion relation is $\Omega^{2}+\kappa^{2}=1$ for evanescent waves (due to the change of space variable, we have $\kappa=c \lambda$ where $\lambda$ is defined in (7)).

Varying the position $x_{0}$, the above expression has a maximum value for $x_{0}=x_{m}$ given by $\sinh ^{2} \kappa x_{m}=1+\kappa^{2} / \Omega^{2}$ and the related maximum amplitude of the derivative eventually results as the simple expression

$$
B_{s}=2\left(1-\Omega^{2}\right) \text {. }
$$

This is the threshold prediction for the Neumann condition (38) and we will now check it with numerical simulations.

As in section 2, we solve the discrete system (34) with no constant bias $(I=0)$, without damping in a first stage $(\gamma=0)$, for a chain of 60 particles with an absorbing boundary on the last 30, and with a coupling factor $c^{2}=25$. The result is displayed in figure 15 which shows good agreement except perhaps around the frequencies 0.33 and 0.18 as in figure 4 (for the same reasons) and close to the phonon band, which is due to the absorbing end.

\subsection{Josephson transmission line (JTL)}

Above, the situation is that of a quasi-continuous undamped and semi-infinite sine-Gordon chain subjected to Neumann condition (38) at the origin. We turn back now to the discrete case, a one-dimensional finite-length (open-ended) array of coupled short Josephson junctions, where the first pendulum is subjected to an external AC driving.

That is, we consider the JTL system

$$
\begin{aligned}
& \ddot{u}_{1}+\gamma \dot{u}_{1}+\sin u_{1}=c^{2}\left(u_{2}-u_{1}\right)+I_{0}+I \sin \Omega t, \\
& \ddot{u}_{n}+\gamma \dot{u}_{n}+\sin u_{n}=I_{0}+c^{2}\left(u_{n+1}-2 u_{n}+u_{n-1}\right), \\
& u_{N+1}=u_{N} .
\end{aligned}
$$

Nonlinear supratransmission becomes here the property of the JTL of transmitting energy in the form of kinks (or antikinks) as soon as the intensity $I$ of the AC driving of the first junction exceeds the threshold

$$
I_{s}=2 c\left(1-\Omega^{2}\right)
$$

as given by the definition $I=-c B$ and from expression (40).

A new fact here is that, for Neumann type boundary conditions such as (36), only kinks (or antikinks) are produced, not breathers. This is the case because producing a kink costs half of the energy required to produce a breather, and because of the freedom left for the boundary value $u_{0}(t)$ (only the difference $u_{1}-u_{0}$ is prescribed), allowing full $2 \pi$ rotations. For the Dirichlet boundary condition as in preceding sections, the prescription for $u_{0}(t)$, e.g. from (2), prevents full rotation.

To illustrate this property, we show in figure 16 a typical simulation with the following set of parameters:

$$
N=10, \quad \gamma=0.1, \quad c=3, \quad I_{0}=0.1,
$$

constituting a reasonable choice for an experimental situation. At frequency $\Omega=0.7$, the threshold (44) is $I_{s}=3.06$, and for an AC driving at amplitude $I=3.3$ the chain starts to rotate, which means that a number of kinks (elementary $2 \pi$ rotation) are generated by the AC 


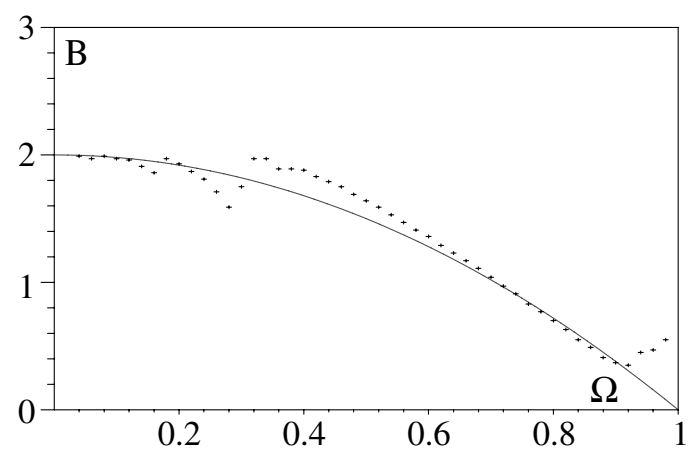

Figure 15. The bifurcation diagram in the $(B, \Omega)$ plane. The solid curve is the threshold expression (40). The crosses indicate the lowest value of $B$ for which nonlinear supratransmission occurs.

driving. In the case of figure 16 about 184 kinks have been generated during 1000 units of normalized time.

Note that we have assumed a small constant-bias DC current $I_{0}=0.1$ in order to select kinks instead of possible antikinks. However, the process does work without bias, although in some of the experiments the rotation would stop after some time (for a reason that we do not understand). Finally, we mention that we have selected compatible boundary conditions: first, an AC forcing that starts from zero and slowly reaches the value $I$ within 100 units of time; and, second, the following initial positions and velocities:

$$
u_{n}(0)=\arcsin \left(I_{0}\right), \quad \dot{u}_{n}(0)=0 .
$$

Then the nonlinear supratransmission does work in such a JTL, despite the small number of oscillators, the presence of damping, the free-end boundary condition, and the presence of constant bias.

\subsection{Energy transmission}

In order to compute the transmitted energy in the present situation, we start with expression (21) but written for a finite number $N$ of oscillators, i.e.

$$
E=\sum_{n=1}^{N} H_{n}+\frac{c^{2}}{2}\left(u_{1}-u_{0}\right)^{2} .
$$

As before, this expression is differentiated and, by use of the conservation law (19), the free boundary condition $u_{N+1}=u_{N}$ leads to the same result, namely

$$
\frac{\partial}{\partial t} E=c^{2} \dot{u}_{0}\left(u_{0}-u_{1}\right) \text {. }
$$

Now, to compute the total energy injected into the medium during time $T$, it is necessary to use integration by parts together with the Neumann condition (36) and to chose for $T$ an integer multiple of the period of the driving. We eventually obtain $(\ell=20$ in the numerical simulations)

$$
T=\ell \frac{2 \pi}{\omega}, \quad E=-I \Omega \int_{0}^{T} \mathrm{~d} t u_{1}(t) \cos \Omega t .
$$

This is the quantity that we have computed, for each value of the AC driving $I$, in figure 17 . 




Figure 16. The motion of particle 5 in a JTL of ten junctions with the parameters (45).

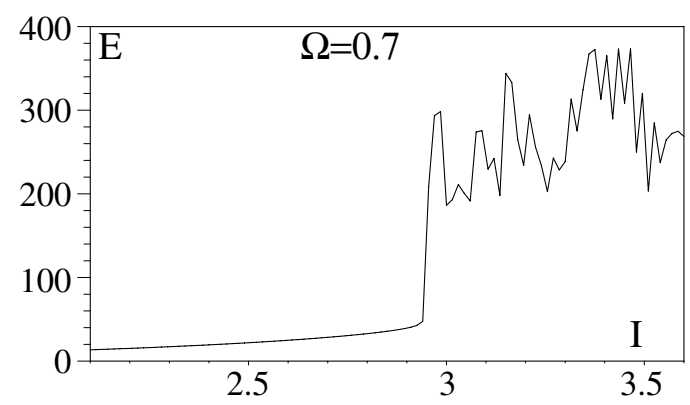

Figure 17. The energy transmitted for 180 time units by a JTL in terms of the driving intensity $I$.

The bifurcation of energy transmission is now quite clear on the graph of the energy $E(T)$ transmitted to the chain displayed in figure 17 obtained for $\Omega=0.7$ and the parameters given in (45).

\section{Conclusions}

The ability of a nonlinear medium to transmit energy when subjected to periodic boundary driving at a frequency in a stop gap and at an amplitude beyond a threshold value, which we called nonlinear supratransmission, has been shown to have some universality (so far, in the domain of nonlinear wave-type equations). The process does not rely on integrability and is robust against damping, discreteness (in a reasonable range), finiteness, and different classes of boundary values. The mechanism at the origin of this process is a nonlinear instability which is still under study.

Moreover, the nonlinear supratransmission has a simple experimental realization in the chain of pendulums, which works surprisingly well. Some other experimental results are expected for JTLs.

\section{Acknowledgments}

It is a pleasure to acknowledge enlightening discussions with M J Ablowitz, M Remoissenet, and A V Ustinov, and decisive experimental help from N Clementin, O Guille, P Munier, and Y Patin. 


\section{References}

[1] Fermi E, Pasta J R and Ulam S M 1955 Studies of nonlinear problems Los Alamos Science Laboratory Report LA-1940

reproduced in

Newell A C (ed) 1974 Nonlinear Wave Motion (AMS Lectures on Applied Mathematics vol 15) (New York: American Mathematical Society)

[2] Gardner C S, Greene J M, Kruskal M D and Miura R M 1967 Phys. Rev. Lett. 191095

[3] Zakharov V E and Shabat A B 1972 Sov. Phys.-JETP 3762

[4] Ablowitz M J, Kaup D J, Newell A C and Segur H 1974 Stud. Appl. Math. 53249

[5] Remoissenet M 1999 Waves Called Solitons (Berlin: Springer)

Scott A C 1999 Nonlinear Science (New York: Oxford University Press)

[6] Geniet F and Leon J 2002 Phys. Rev. Lett. 89134102

[7] Caputo J-G, Leon J and Spire A 2001 Phys. Lett. A 283 129-35

[8] Barone A and Paternó G 1982 Physics and Application of the Josephson Effect (New York: Wiley) Ustinov A V 1998 Physica D 123315

[9] van der Zant H S J, Barahona M, Duwel A E, Orlando T P, Watanabe S and Strogatz S 1998 Physica D 119219

[10] Floria L M, Marin J L, Martinez P J, Falo F and Aubry S 1996 Europhys. Lett. 36539

Flach S and Spicci M 1999 J. Phys.: Condens. Matter 11321

[11] Trias E, Mazo J J and Orlando T P 2000 Phys. Rev. Lett. 84741

Binder P, Abraimov D, Ustinov A V, Flach S and Zolotaryuk Y 2000 Phys. Rev. Lett. 84745

[12] Ustinov A V, Mygind J and Oboznov V A 1992 J. Appl. Phys. 721203

Ustinov A V, Mygind J, Pedersen N F and Oboznov V A 1992 Phys. Rev. B 46578 